\title{
Investigating the Activation Kinetics of Phosphoramidites for Oligonucleotide Synthesis
}

Fiona J. Laraman*, $\dagger$, Heidi Fisk ${ }^{\dagger}$, David T. E. Whittaker ${ }^{\ddagger}$, Janette H. Cherryman ${ }^{\dagger}$, Louis J. Diorazio ${ }^{\dagger}$

${ }^{\dagger}$ Chemical Development, Pharmaceutical Technology \& Development, Operations, AstraZeneca, Macclesfield, SK10 2NA, UK

‡Early Chemical Development, Pharmaceutical Sciences, BioPharmaceuticals R\&D, AstraZeneca, Macclesfield, SK10 2NA, UK

Contents

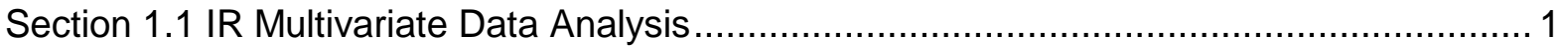

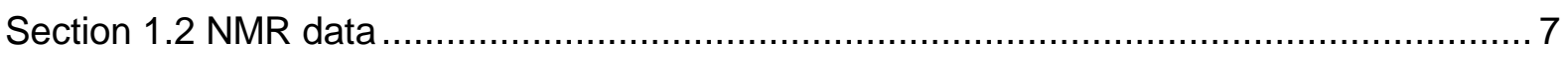

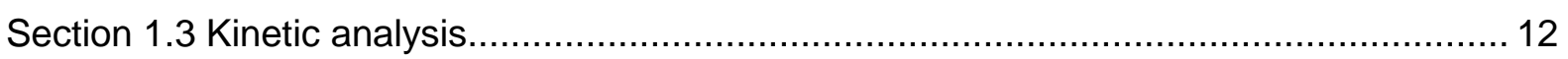

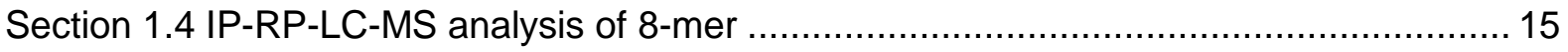

Section 1.1 IR Multivariate Data Analysis

Batch Activation Experiments: In-line IR spectra were acquired utilising a Mettler Toledo ReactIR 701L spectrometer coupled to an ATR DiComp AgX 6 mm x $1.5 \mathrm{~m}$ fibre immersion probe. Mettler Toledo iC IR software version 7.1 was used with fast scanning enabled to allow for rapid acquisition. Ensuring that the ATR tip was immersed in the agitated phosphoramidite solution, IR data acquisition commenced collecting spectra at a rate of every 3 seconds using 16 scans, $1 \times$ gain and $8 \mathrm{~cm}^{-1}$ resolution. The activator solution was then rapidly charged in one portion and IR data acquisition occurred for approximately 5 mins for a DNA phosphoramidite and 15 mins for a cEt phosphoramidite. IR experiments were performed 
in duplicate for all of the phosphoramidites and conditions mentioned within this publication. Note, experiments were performed under stringent anhydrous conditions due to the innate sensitivity of IR to water and to avoid undesirable hydrolysis reactions.

Multivariate analysis to derive activation trends was performed post acquisition using functions available within the PLS toolbox version 8.8.1. IR spectra were pre-processed by removing any spectra acquired before activator addition, followed by truncating the spectral region to $1632-680 \mathrm{~cm}^{-1}$ then applying a $1^{\text {st }}$ derivative (11 pt) and mean centring prior to principal component analysis (PCA). PCA is an unsupervised multivariate method, meaning that no prior information is provided to the algorithm. PCA acts to reduce the dimensionality of the data whilst retaining the variance (trends) within the dataset. The main principal component $(P C), P C 1$, explains the most significant trend extracted from the data. PC 1 corresponded to activation events for all phosphoramidites analysed (excluding those experiencing hydrolysis as described below), with PC 1 explaining between $69-94 \%$ of the variance within the dataset. Subsequent PCs explained lower-level variance such as slight concentration differences between duplicate experiments, negligible hydrolysis or innate noise in the data.

To exemplify hydrolysis versus activation events, the following figures (Figures S1 S3) illustrate the differences observed by IR for 16. Figure S1A shows the desired IR spectral response for $\mathbf{1 6}$ due to activation. Comparing this response to that of Figure S1B, which encounters both activation and adverse hydrolysis, it is evident that there are differences between the two responses, most notably between $1100-950 \mathrm{~cm}^{-1}$. 

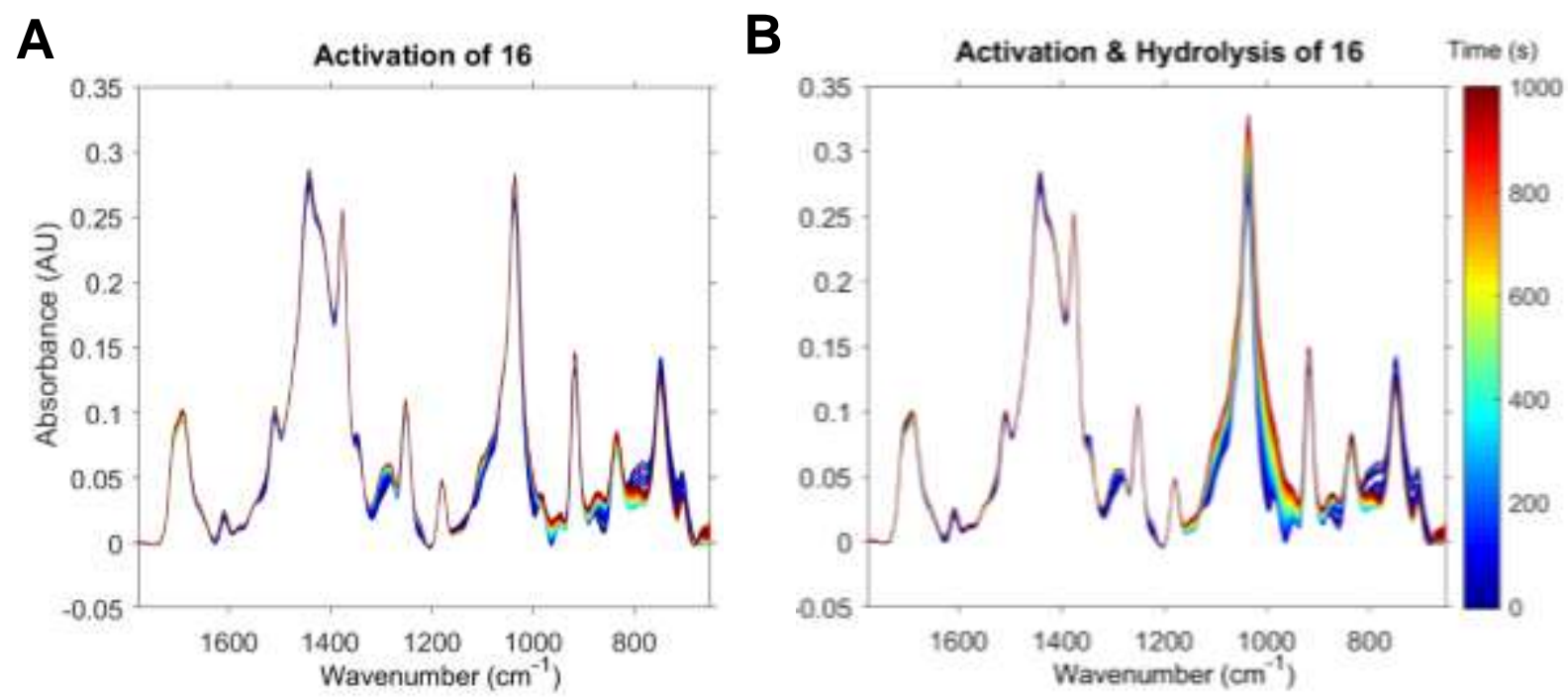

Figure S1. IR spectra for the activation of $16(1: 1 \mathrm{v} / \mathrm{v}, 5 \mathrm{~mol}$ eq of 2$)$ over $1000 \mathrm{~s}$ monitoring period. A) The desired IR response arising from the activation of 16. B) Both activation and hydrolysis. A colour gradient highlights how the response is changing with respect to time.

PCA was performed on the IR datasets; 16 undergoing activation only (A) and $\mathbf{1 6}$ experiencing competing activation and hydrolysis (B) (Figure S2). Pre-processing was consistent with that used for modelling activation events (truncating, $1^{\text {st }}$ derivate and mean centring). Unlike (A), dataset (B) does not plateau succeeding activation events; instead (B) has a linear trajectory between $300-1000$ seconds. This linear response was caused by unwanted moisture ingress resulting in hydrolysis of the activated phosphoramidite. 


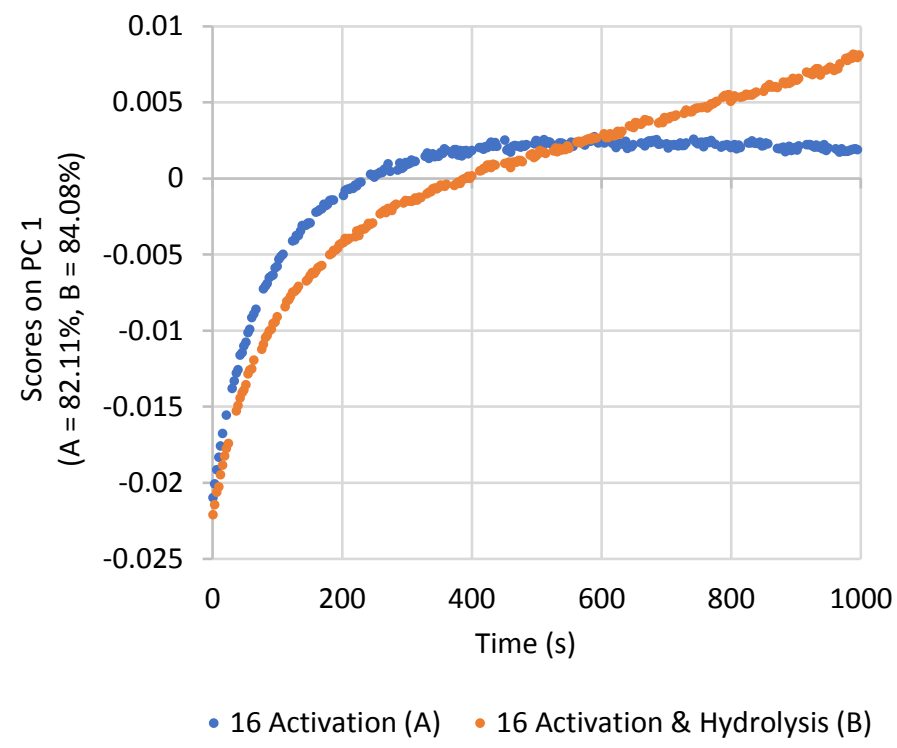

Figure S2. Plot of PC 1 scores versus time (s) for the activation of 16. Blue = the desired trend arising from activation (A), with $P C 1$ explaining $82 \%$ variance. Orange = the activation and competing hydrolysis of $16(B)$, with $P C 1$ explaining $84 \%$ variance. PCA was performed independently on the two datasets; the data has been combined onto the same $y$-axis for illustrative purposes.

By comparing the PC 1 loadings in Figure S3, this further illustrates that the dominant features in the IR response which are attributable to hydrolysis (B) occur between $1100-950 \mathrm{~cm}^{-1}$. Peak changes associated to activation (Figure S3A) are still detectable amongst the hydrolysis (Figure S3B), however deconvoluting these two events will become increasingly difficult if high levels of hydrolysis occur. Subsequent offline liquid chromatography-mass spectrometry (LCMS) analysis determined that only low-level hydrolysis to the unreactive $\mathrm{H}$-phosphonate 18 had occurred in (B). However, this still had a noticeable impact on the IR response, demonstrating the innate sensitivity to this adverse event. Therefore, it is imperative to ensure rigorous anhydrous conditions so that only activation is monitored. 

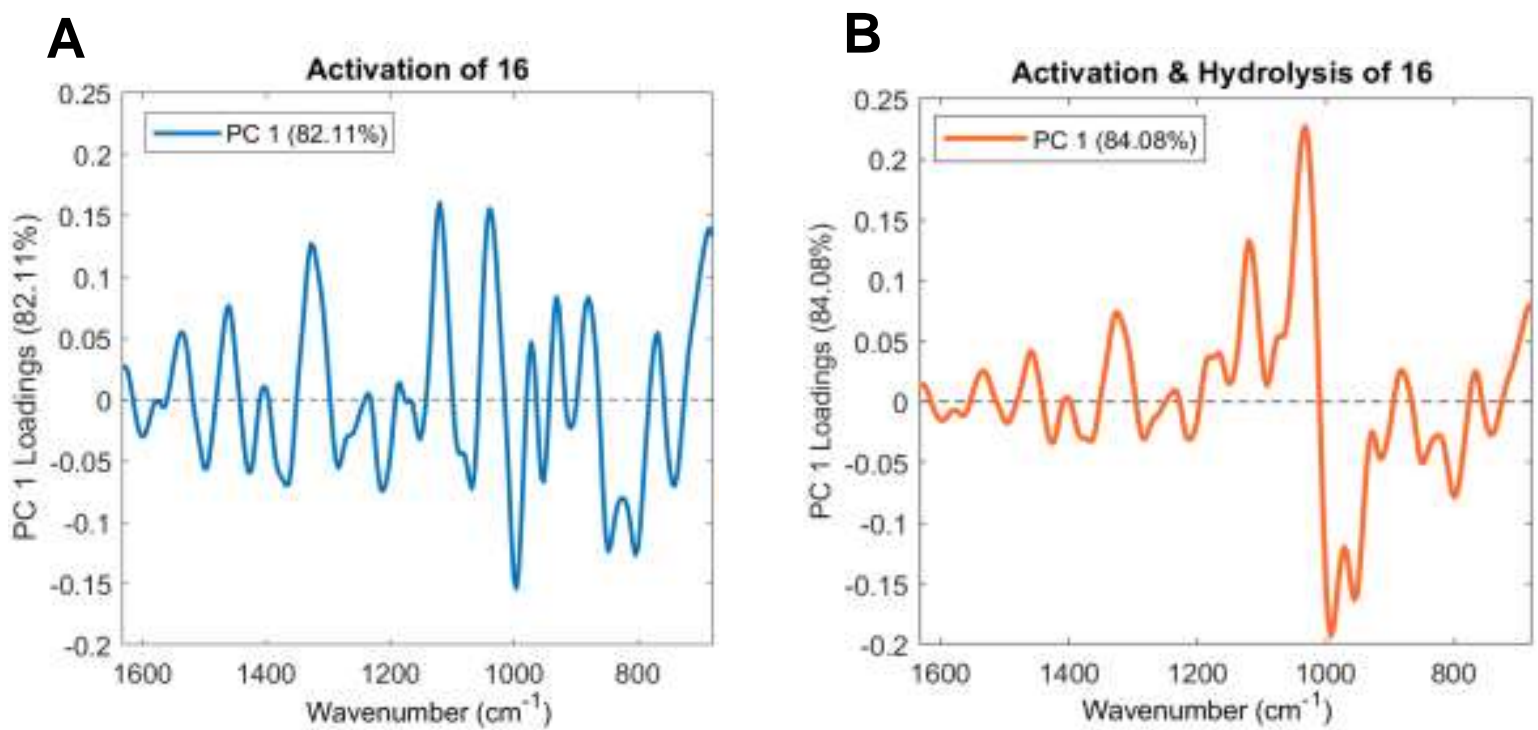

Figure S3. Plot of PC 1 loadings of PCA. A) Loadings of the activation of 16. B) Loadings which include competing hydrolysis and activation of 16. There are key similarities between the responses, however hydrolysis gives rise to dominant spectral changes between $1100-950 \mathrm{~cm}^{-1}$.

Figure $S 4$ is a plot of known reference spectra involved in the activation of 16 , spectra correlating to the start of activation (once activator has been added) and spectra at the end (no further change in IR response and activation has ceased). The concentrations are representative of reaction conditions for the $1: 1 \mathrm{v} / \mathrm{v}$ activator solution $(1 \mathrm{M} \mathrm{2}, 0.1 \mathrm{M} \mathrm{NMI})$ to phosphoramidite 16 solution $(0.2 \mathrm{M})$. The diisopropylamine 7 spectrum is representative of $100 \%$ activation of $\mathbf{1 6}$. It is evident that this response closely resembles that $\mathrm{MeCN}$, identifying that the IR response is very weak and will go undetected. Comparing the start and end responses with the known reference spectra in Figure S4, the peaks experiencing change on activation are peaks associated to either the phosphoramidite $\mathbf{1 6}$ or the activator response, as expected. 


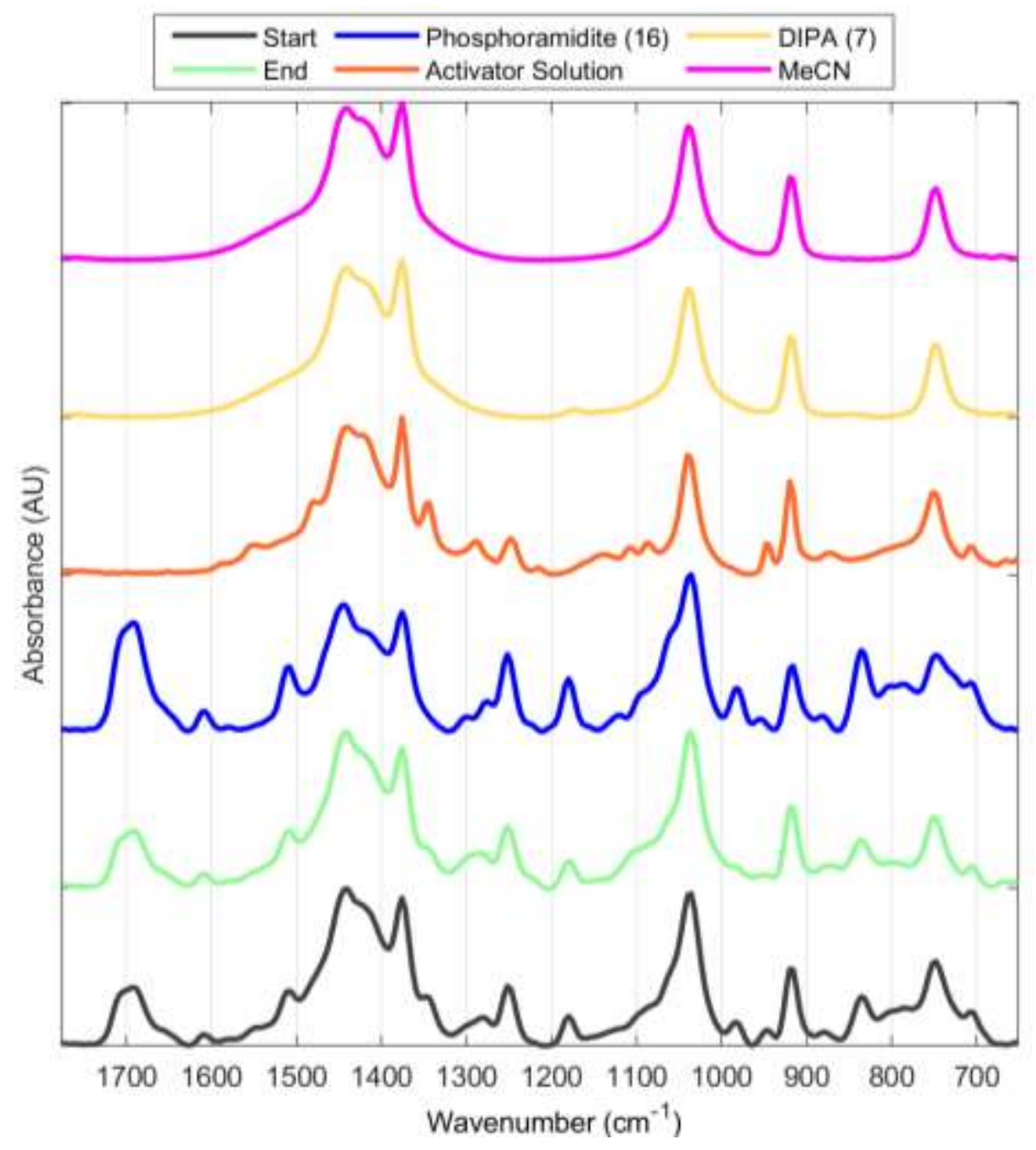

Figure S4. IR reference spectra of reaction components for the activation of 16 . The IR responses are of concentrations representative of reaction conditions. The start and end spectra refer to the start of activation (once activator solution has been added) and the end is once there is no further change detectable in the IR response (activation complete/stopped).

To determine if the activation trends derived from IR analysis agreed with quantitative NMR analysis, the resultant profiles for the two techniques were overlaid to enable comparisons. The PC 1 scores derived from PCA of the IR data are plotted against time (s), depicting the rate of activation for 9 and 10 (Figure S5, Figure S6 respectively). NMR trends studying the liberation of diisopropylamine 7 and hence the percentage of activation (\%) have been included. Evidently these two techniques are in excellent agreement and corroborate the rate of activation. 


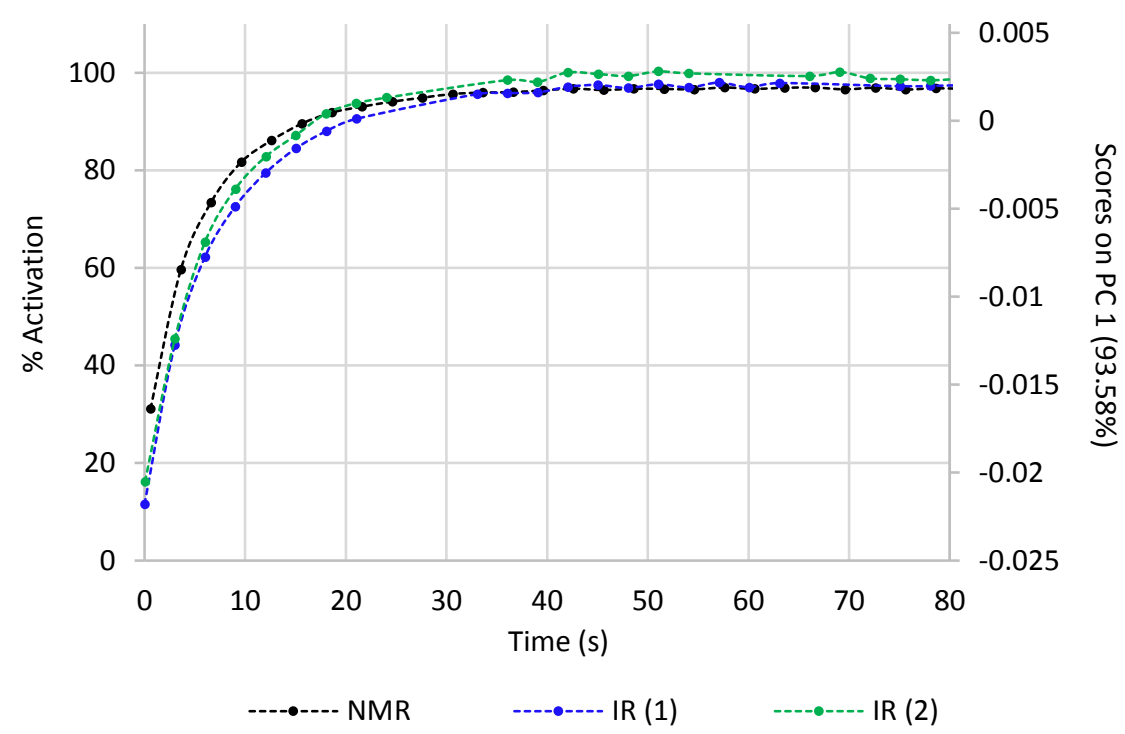

Figure S5: NMR and IR trends for activation of 9 (5 eq 2).

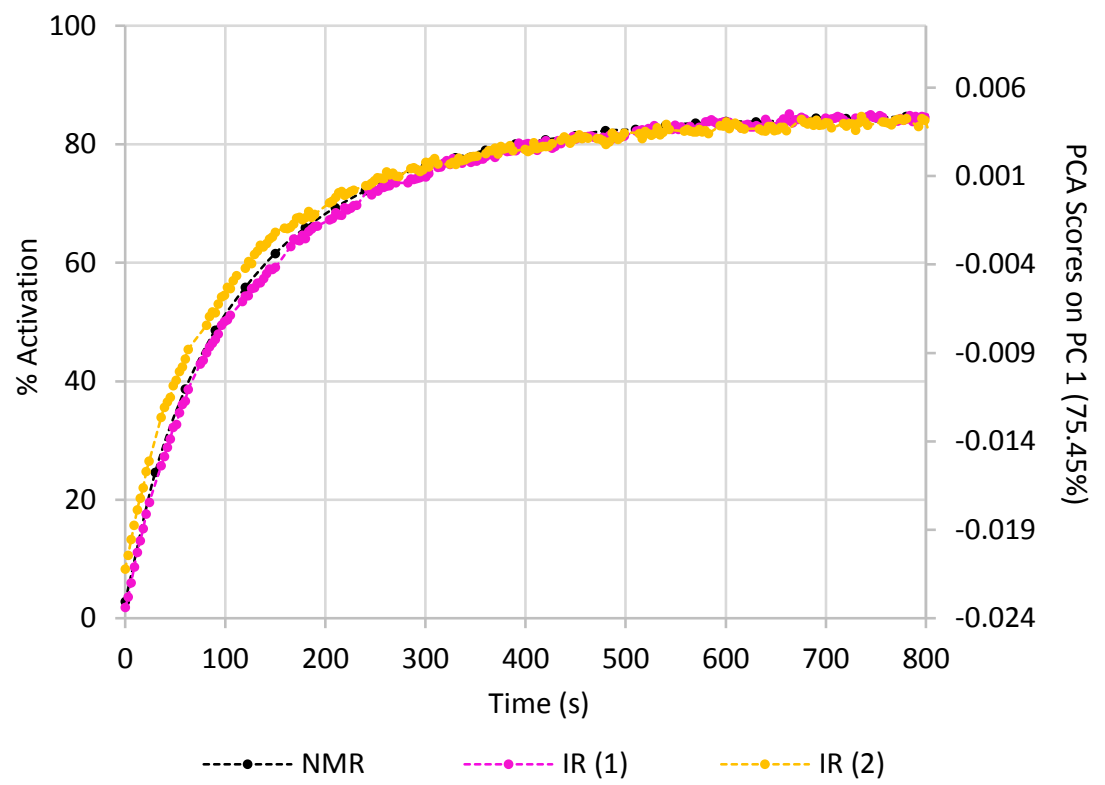

Figure S6: NMR and IR trends for activation of 10 (5 eq 2).

\section{Section 1.2 NMR data}

All NMR data was recorded on a Bruker AVIIIHD spectrometer fitted with a BBFO probe with an operating frequency of $500.13 \mathrm{MHz}$ for proton. The InsightXpress platform operates using Topspin v3.6.0 software and all ${ }^{1} \mathrm{H}$ data were acquired using WET suppression of the solvent signal with simultaneous decoupling of ${ }^{13} \mathrm{C}$ to remove satellites. All data were acquired using 
a $15^{\circ}$ pulse and an acquisition time of 1.64 seconds to ensure that quantitative information was obtained from each single scan experiment.

NMR data were stacked and integrated en masse using Mestrenova v12.0.1 software and extracted integral values were further processed using Microsoft Excel.

Spectra for all DNA phosphoramidite activations were acquired immediately after reaction initiation and every 3 seconds thereafter. In order to obtain an accurate time for the initial data point, the first two integral values for the diisopropylamine signal were plotted against time and the plot axis adjusted to achieve a y axis intercept at 0 . This gave an initial time point of 0.6 seconds which was then applied to all DNA phosphoramidite plots.

The first two integral values for the unactivated phosphoramidite signal at $0.94 \mathrm{ppm}$ were then plotted against this adjusted timescale to give a y axis intercept representing the integral value at $t=0$. This initial integral value for unactivated phosphoramidite, along with a latter once the reaction had plateaued, were used to determine the overall \% activation of the phosphoramidite. The integral values for the diisopropylamine signal could then be converted to represent $\%$ activation vs time.

Spectra for all cEt phosphoramidite activations were acquired immediately after reaction initiation and every 30 seconds thereafter. The 0.6 second instrument switching delay was considered negligible on this timescale and so initial data points were quoted as occurring at 0 seconds in all cases. Likewise, \% activation calculations were based on the initial integral value recorded for the unactivated phosphoramidite along with a latter once the reaction had plateaued.

Table S1: \% Activation vs Time data for all DNA phosphoramidites (5 eq 2).

\begin{tabular}{ccccc}
\hline Time (s) & $\mathbf{9}$ & $\mathbf{1 1}$ & $\mathbf{1 3}$ & $\mathbf{1 5}$ \\
\hline $\mathbf{0}$ & & & & \\
$\mathbf{0 . 6}$ & 31.11 & 9.23 & 7.72 & 9.82 \\
$\mathbf{3 . 6}$ & 59.63 & 49.80 & 46.08 & 54.11 \\
$\mathbf{6 . 6}$ & 73.42 & 69.47 & 63.77 & 71.90 \\
\hline
\end{tabular}




\begin{tabular}{|c|c|c|c|c|}
\hline 9.6 & 81.67 & 80.54 & 73.80 & 81.30 \\
\hline 12.6 & 86.13 & 87.47 & 80.15 & 86.72 \\
\hline 15.6 & 89.55 & 91.75 & 84.45 & 90.05 \\
\hline 18.6 & 91.85 & 94.62 & 87.34 & 92.19 \\
\hline 21.6 & 93.06 & 96.61 & 89.46 & 93.65 \\
\hline 24.6 & 94.09 & 97.74 & 90.85 & 94.44 \\
\hline 27.6 & 94.89 & 98.72 & 91.99 & 95.06 \\
\hline 30.6 & 95.66 & 99.16 & 92.66 & 95.57 \\
\hline 33.6 & 95.95 & 99.47 & 93.19 & 95.69 \\
\hline 36.6 & 96.04 & 99.89 & 93.65 & 96.02 \\
\hline 39.6 & 96.39 & 99.92 & 93.87 & 95.92 \\
\hline 42.6 & 96.74 & 100.05 & 94.22 & 96.12 \\
\hline 45.6 & 96.48 & 100.13 & 94.42 & 96.18 \\
\hline 48.6 & 96.74 & 100.14 & 94.46 & 96.11 \\
\hline 51.6 & 96.70 & 100.15 & 94.51 & 96.13 \\
\hline 55.6 & 96.62 & 100.32 & 94.50 & 96.25 \\
\hline 57.6 & 96.98 & 100.27 & 94.52 & 96.15 \\
\hline 60.6 & 96.71 & 100.32 & 94.59 & 96.35 \\
\hline 63.6 & 96.93 & 100.18 & 94.74 & 96.04 \\
\hline 66.6 & 96.97 & 100.26 & 94.73 & 96.06 \\
\hline 69.6 & 96.62 & 100.15 & 94.80 & 96.34 \\
\hline 72.6 & 96.94 & 100.28 & 94.75 & 96.24 \\
\hline 75.6 & 96.58 & 100.20 & 94.77 & 96.12 \\
\hline 78.6 & 96.83 & 100.30 & 94.68 & 96.30 \\
\hline 81.6 & 96.69 & 100.34 & 94.65 & 96.30 \\
\hline 84.6 & 96.58 & 100.26 & 94.78 & 96.17 \\
\hline 87.6 & 96.89 & 100.29 & 94.78 & 96.10 \\
\hline
\end{tabular}


Table S2: \% Activation vs Time data for all cEt phosphoramidites (5 eq 2).

Time (s)

0

30

60

90

120

150

180

210

240

270

300

330

360

390

420

450

480

510

540

570

600

630
12

14

3.11

18.08

29.39

38.32

45.03

50.75

55.53

59.20

62.46

65.03

67.41

69.53

71.12

72.72

74.20

75.31

76.35

77.31

78.01

78.65

79.39

79.73
16

10
2.63

2.81

25.25

39.74

49.28

56.24

61.48

65.47

68.77

71.62

73.39

75.05

76.31

77.44

78.25

79.20

79.67

80.05

80.59

81.27

81.32

81.41

81.47
24.61

38.67

48.64

55.84

61.60

65.90

69.32

72.16

74.55

76.24

77.74

78.97

80.05

80.77

81.47

82.29

82.51

83.15

83.58

83.85

83.79 


\begin{tabular}{lllll}
\hline 660 & 80.17 & 80.16 & 81.83 & 84.05 \\
690 & 80.45 & 80.76 & 81.92 & 84.44 \\
720 & 81.07 & 81.18 & 82.00 & 84.33 \\
750 & 81.54 & 81.35 & 82.09 & 84.47 \\
780 & 81.76 & 81.78 & 82.33 & 84.67 \\
810 & 81.96 & 82.15 & 82.52 & 84.80
\end{tabular}

Table S3: \% Activation vs Time data for all cEt phosphoramidites (11 eq 2).

\begin{tabular}{|c|c|c|c|c|}
\hline Time (s) & 12 & 14 & 16 & 10 \\
\hline 0 & 1.80 & 3.92 & 4.85 & 4.13 \\
\hline 30 & 32.04 & 37.43 & 44.38 & 40.61 \\
\hline 60 & 52.52 & 58.86 & 65.30 & 60.73 \\
\hline 90 & 66.12 & 71.93 & 77.65 & 72.97 \\
\hline 120 & 75.25 & 80.83 & 85.15 & 80.71 \\
\hline 150 & 82.31 & 86.65 & 90.13 & 85.89 \\
\hline 180 & 86.62 & 90.35 & 93.07 & 89.40 \\
\hline 210 & 89.76 & 93.13 & 95.26 & 92.01 \\
\hline 240 & 92.36 & 95.03 & 96.67 & 93.74 \\
\hline 270 & 94.15 & 96.24 & 97.61 & 95.05 \\
\hline 300 & 95.73 & 97.24 & 98.04 & 95.89 \\
\hline 330 & 96.66 & 97.97 & 98.42 & 96.59 \\
\hline 360 & 97.41 & 98.37 & 99.05 & 97.33 \\
\hline 390 & 97.97 & 98.84 & 99.26 & 97.68 \\
\hline 420 & 98.46 & 99.11 & 99.28 & 97.85 \\
\hline 450 & 98.59 & 99.29 & 99.29 & 98.11 \\
\hline 480 & 99.08 & 99.37 & 99.42 & 98.36 \\
\hline 510 & 99.18 & 99.32 & 99.52 & 98.50 \\
\hline
\end{tabular}




\begin{tabular}{lllll}
\hline $\mathbf{5 4 0}$ & 99.45 & 99.45 & 99.71 & 98.46 \\
$\mathbf{5 7 0}$ & 99.28 & 99.65 & 99.59 & 98.60 \\
$\mathbf{6 0 0}$ & 99.68 & 99.67 & 99.59 & 98.71 \\
$\mathbf{6 3 0}$ & 99.34 & 99.54 & 99.77 & 98.71 \\
$\mathbf{6 6 0}$ & 99.65 & 99.45 & 99.64 & 98.78 \\
$\mathbf{6 9 0}$ & 99.62 & 99.69 & 99.73 & 98.94 \\
$\mathbf{7 2 0}$ & 99.99 & 99.67 & 99.74 & 99.00 \\
$\mathbf{7 5 0}$ & 99.77 & 99.76 & 99.59 & 98.79 \\
$\mathbf{7 8 0}$ & 99.79 & 99.61 & 99.58 & 98.95 \\
$\mathbf{8 1 0}$ & 99.61 & 99.54 & 99.58 & 98.97 \\
\hline
\end{tabular}

\section{Section 1.3 Kinetic analysis}

Processed data was received from the IR and NMR analytical work. In order to extract the pseudo first order rate constant this data was converted into \% activation assuming complete conversion when reaction data plateaued. This conversion data was then fitted using Dynochem to extract the pseudo first order rate constant using the expression:

Phosphoramidite $\mathbf{1} \rightarrow$ Activated phosphoramidite $\mathbf{5}+$ diisopropylamine $\mathbf{7}$

2 is in large excess and it can therefore be assumed its concentration does not change as the reaction progresses, hence we can treat this reaction as first order in 1. Each set of data was processed individually, resulting in a formation rate constant for each experiment which can then be compared to give a quantitative evaluation of the two results measured by IR (IR1 and IR2) and compared to NMR.

Table S4: Full kinetic data set.

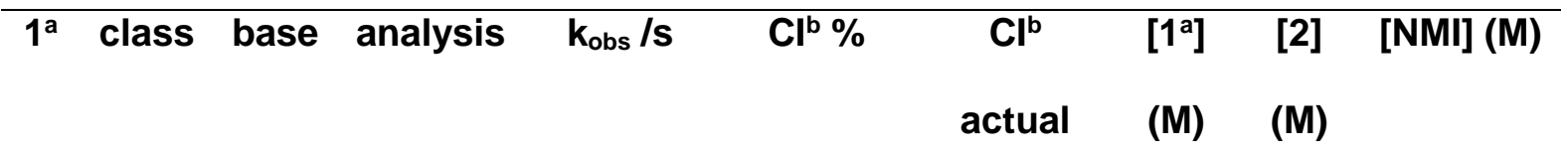




\begin{tabular}{|c|c|c|c|c|c|c|c|c|c|}
\hline 9 & DNA & $A$ & NMR & 1.47E-01 & $3.76 \mathrm{E}+00$ & 5.53E-03 & 0.10 & 0.50 & 0.05 \\
\hline 9 & DNA & $A$ & IR1 & 1.39E-01 & 4.47E+00 & $6.20 \mathrm{E}-03$ & 0.10 & 0.50 & 0.05 \\
\hline 9 & DNA & $A$ & IR2 & 1.39E-01 & $3.41 \mathrm{E}+00$ & 4.73E-03 & 0.10 & 0.50 & 0.05 \\
\hline 10 & cEt & $A$ & NMR & $1.44 \mathrm{E}-02$ & $4.40 \mathrm{E}+00$ & 6.34E-04 & 0.06 & 0.70 & 0.07 \\
\hline 10 & cEt & $A$ & IR1 & 1.68E-02 & $1.13 E+00$ & 1.90E-04 & 0.06 & 0.70 & 0.07 \\
\hline 10 & cEt & $A$ & IR2 & 1.48E-02 & $1.25 \mathrm{E}+00$ & 1.85E-04 & 0.06 & 0.70 & 0.07 \\
\hline 10 & cEt & $A$ & NMR & $5.60 \mathrm{E}-03$ & $5.00 \mathrm{E}+00$ & 2.80E-04 & 0.10 & 0.50 & 0.05 \\
\hline 10 & cEt & $A$ & IR1 & $5.20 \mathrm{E}-03$ & 9.01E-01 & 4.70E-05 & 0.10 & 0.50 & 0.05 \\
\hline 10 & cEt & $A$ & IR2 & 5.90E-03 & 8.54E-01 & 5.00E-05 & 0.10 & 0.50 & 0.05 \\
\hline 11 & DNA & $\mathrm{MeC}$ & NMR & $1.60 \mathrm{E}-01$ & $6.66 \mathrm{E}+00$ & 1.07E-02 & 0.10 & 0.50 & 0.05 \\
\hline 11 & DNA & $\mathrm{MeC}$ & IR1 & $1.45 \mathrm{E}-01$ & $5.49 E+00$ & 7.94E-03 & 0.10 & 0.50 & 0.05 \\
\hline 11 & DNA & $\mathrm{MeC}$ & IR2 & 1.64E-01 & 7.52E+00 & 1.23E-02 & 0.10 & 0.50 & 0.05 \\
\hline 12 & cEt & $\mathrm{MeC}$ & NMR & 5.40E-03 & $5.24 \mathrm{E}+00$ & 2.83E-04 & 0.10 & 0.50 & 0.05 \\
\hline 12 & cEt & $\mathrm{MeC}$ & IR1 & 6.40E-03 & $1.09 E+00$ & 7.00E-05 & 0.10 & 0.50 & 0.05 \\
\hline 12 & cEt & $\mathrm{MeC}$ & IR2 & 7.80E-03 & 9.54E-01 & 7.40E-05 & 0.10 & 0.50 & 0.05 \\
\hline 12 & cEt & $\mathrm{MeC}$ & NMR & 1.16E-02 & $2.16 \mathrm{E}+00$ & 2.51E-04 & 0.06 & 0.70 & 0.07 \\
\hline 12 & cEt & $\mathrm{MeC}$ & IR1 & 1.06E-02 & $1.55 \mathrm{E}+00$ & 1.64E-04 & 0.06 & 0.70 & 0.07 \\
\hline 12 & cEt & $\mathrm{MeC}$ & IR2 & $1.21 \mathrm{E}-02$ & $2.76 \mathrm{E}+00$ & 3.34E-04 & 0.06 & 0.70 & 0.07 \\
\hline 13 & DNA & $\mathrm{G}$ & NMR & $1.71 \mathrm{E}-01$ & $4.62 \mathrm{E}+00$ & 7.91E-03 & 0.10 & 0.50 & 0.05 \\
\hline 13 & DNA & $\mathrm{G}$ & IR1 & 1.87E-01 & $2.80 \mathrm{E}+00$ & 5.24E-03 & 0.10 & 0.50 & 0.05 \\
\hline 13 & DNA & $\mathrm{G}$ & IR2 & $1.64 \mathrm{E}-01$ & $6.32 \mathrm{E}+00$ & 1.03E-02 & 0.10 & 0.50 & 0.05 \\
\hline 14 & cEt & $\mathrm{G}$ & NMR & 1.37E-02 & $2.05 \mathrm{E}+00$ & 2.80E-04 & 0.06 & 0.70 & 0.07 \\
\hline 14 & cEt & $\mathrm{G}$ & IR1 & $1.20 \mathrm{E}-02$ & $2.11 \mathrm{E}+00$ & 2.53E-04 & 0.06 & 0.70 & 0.07 \\
\hline 14 & cEt & $\mathrm{G}$ & IR2 & 1.13E-02 & $2.29 \mathrm{E}+00$ & 2.59E-04 & 0.06 & 0.70 & 0.07 \\
\hline 14 & cEt & $\mathrm{G}$ & NMR & $5.60 \mathrm{E}-03$ & $5.00 \mathrm{E}+00$ & 2.80E-04 & 0.10 & 0.50 & 0.05 \\
\hline 14 & cEt & $\mathrm{G}$ & IR1 & 5.20E-03 & 9.01E-01 & 4.70E-05 & 0.10 & 0.50 & 0.05 \\
\hline 14 & cEt & G & IR2 & 5.90E-03 & 8.54E-01 & 5.00E-05 & 0.10 & 0.50 & 0.05 \\
\hline
\end{tabular}




\begin{tabular}{lccccccccc}
15 & DNA & T & NMR & $2.03 \mathrm{E}-01$ & $6.81 \mathrm{E}+00$ & $1.38 \mathrm{E}-02$ & 0.10 & 0.50 & 0.05 \\
15 & DNA & $\mathrm{T}$ & $\mathrm{IR} 1$ & $1.99 \mathrm{E}-01$ & $8.38 \mathrm{E}+00$ & $1.67 \mathrm{E}-02$ & 0.10 & 0.50 & 0.05 \\
15 & $\mathrm{DNA}$ & $\mathrm{T}$ & $\mathrm{IR} 2$ & $2.02 \mathrm{E}-01$ & $1.05 \mathrm{E}+01$ & $2.12 \mathrm{E}-02$ & 0.10 & 0.50 & 0.05 \\
16 & $\mathrm{cEt}$ & $\mathrm{MeU}$ & $\mathrm{NMR}$ & $1.64 \mathrm{E}-02$ & $3.06 \mathrm{E}+00$ & $5.02 \mathrm{E}-04$ & 0.06 & 0.70 & 0.07 \\
16 & $\mathrm{cEt}$ & $\mathrm{MeU}$ & $\mathrm{IR} 1$ & $1.58 \mathrm{E}-02$ & $1.70 \mathrm{E}+00$ & $2.69 \mathrm{E}-04$ & 0.06 & 0.70 & 0.07 \\
16 & $\mathrm{cEt}$ & $\mathrm{MeU}$ & $\mathrm{IR} 2$ & $1.47 \mathrm{E}-02$ & $1.12 \mathrm{E}+00$ & $1.65 \mathrm{E}-04$ & 0.06 & 0.70 & 0.07 \\
16 & $\mathrm{cEt}$ & $\mathrm{MeU}$ & $\mathrm{NMR}$ & $9.20 \mathrm{E}-03$ & $5.93 \mathrm{E}+00$ & $5.45 \mathrm{E}-04$ & 0.10 & 0.50 & 0.05 \\
16 & $\mathrm{cEt}$ & $\mathrm{MeU}$ & $\mathrm{IR} 1$ & $1.10 \mathrm{E}-02$ & $1.22 \mathrm{E}+00$ & $1.34 \mathrm{E}-04$ & 0.10 & 0.50 & 0.05 \\
16 & $\mathrm{cEt}$ & $\mathrm{MeU}$ & $\mathrm{IR} 2$ & $9.40 \mathrm{E}-03$ & $1.55 \mathrm{E}+00$ & $1.46 \mathrm{E}-04$ & 0.10 & 0.50 & 0.05 \\
\hline
\end{tabular}

a - indicates the generalised phosphoramidite with specific species as listed.

b $-\mathrm{Cl}=95 \%$ confidence interval

In order to prove that the formation and consumption rates were consistent with each other, 3 data sets were compared. Each data set was fitted independently and then compared.

Table S5: Comparison of formation and consumption rates across 3 phosphoramidites.

\begin{tabular}{|c|c|c|c|c|c|}
\hline $1^{a}$ & Class & Base & Analysis & $k_{\text {obs }} / \mathrm{s}$ & $\mathrm{Cl}^{\mathrm{b}} \%$ \\
\hline 9 & DNA & A & Consumption & 1.79E-01 & 4.21 \\
\hline 9 & DNA & $A$ & Formation & 1.47E-01 & 3.76 \\
\hline 10 & cEt & $A$ & Consumption & 5.60E-03 & 3.68 \\
\hline 10 & cEt & A & Formation & 5.20E-03 & 0.90 \\
\hline 16 & cEt & $\mathrm{MeU}$ & Consumption & 8.30E-03 & 1.80 \\
\hline 16 & $c E t$ & $\mathrm{MeU}$ & Formation & $9.20 \mathrm{E}-03$ & 5.93 \\
\hline
\end{tabular}

In all cases both the formation and consumption rate constants were very similar indicating that there are no significant alternative reaction pathways taking place and that the formation rates measured are representative of the reaction being proposed. 


\section{Section 1.4 IP-RP-LC-MS analysis of 8-mer}

For ion-pair reversed-phase liquid chromatography-mass spectrometry (IP-RP-LC-MS) analysis of the 8-mer, an Agilent 1260 Infinity II SQD HPLC equipped with an ACQUITY PREMIER Oligonucleotide $\mathrm{BEH} \mathrm{C}_{18}$ column at $70{ }^{\circ} \mathrm{C}$ was used. The mobile phases consisted of $\mathrm{A}: 4 \% \mathrm{MeCN}$ in water, $2 \mathrm{mM}$ tributylammonium acetate (TBuAA), $0.4 \mu \mathrm{M}$ ethylenediaminetetraacetic acid (EDTA) and B: $92 \% \mathrm{MeCN}$ in water, $2 \mathrm{mM}$ TBuAA, $0.4 \mu \mathrm{M}$ EDTA. Program gradient elution (time $(\min ) / \%$ B) 0/20, 28/70, 29/20, 35/20 was used with UV detection at $260 \mathrm{~nm}$ and a flow rate of $0.25 \mathrm{~mL} / \mathrm{min}$. The injection volume was $10 \mu \mathrm{L}$ and the autosampler temperature was $5^{\circ} \mathrm{C}$.

The target 8-mer sequence could be resolved from shorter synthetic impurities. Relative quantitation of trace level shortmers could be detected and reported by UV peak area, although 3-mers and shorter were below the limit of detection (Figure S7). Identity of 8-mer and shortmers were confirmed by mass spectrometry. Pleasingly, it was found that the relative abundance of desired 8-mer after SPS followed the expected trend across experiments.

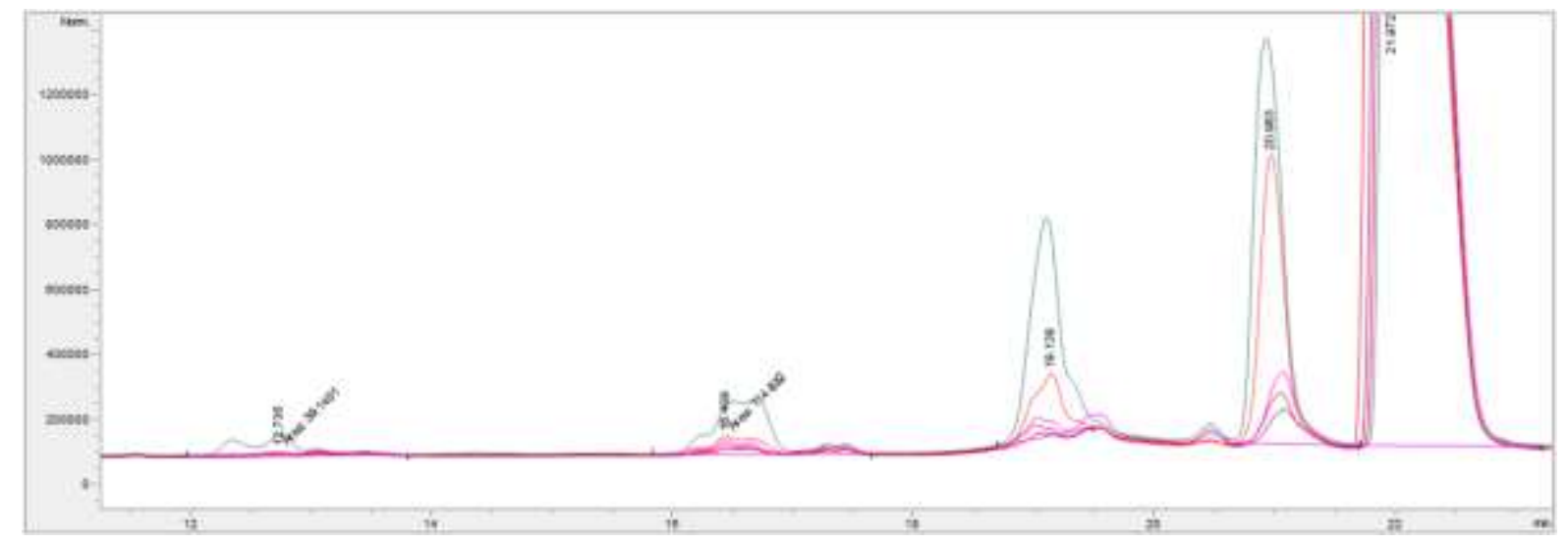

Figure S7: Overlayed UV data for Oligonucleotide synthesis experiments R1-5. The green trace represents R5, followed by the red trace R4 demonstrating increased levels of shortmers for the faster flow rates. Pink and purple traces represent experiments R1-3, with similar levels of impurities. 